\title{
From storytelling to Facebook. Content biases when retelling or sharing a story
}

\section{Author:}

Alberto Acerbi

Address: Centre for Culture and Evolution, Division of Psychology, Brunel University London, Uxbridge, UB8 3PH, United Kingdom

Email: alberto.acerbi@brunel.ac.uk

Orcid ID: 0000-0001-5827-8003

\begin{abstract}
:
Purpose: Cultural evolution researchers use transmission chain experiments to investigate which content is more likely to survive when transmitted from one individual to another. These experiments resemble oral storytelling, where individuals need to understand, memorise, and reproduce the content. However, prominent contemporary forms of cultural transmission - think an online sharing - only involve the willingness to transmit the content. Here I present two fully preregistered online experiments that explicitly investigated the differences between these two modalities of transmission.
\end{abstract}

Methods: The first experiment $(\mathrm{N}=1,080$ participants from $\mathrm{UK})$ examined whether negative content, information eliciting disgust, and threat-related information were better transmitted than their neutral counterpart in a traditional transmission chain set-up. The second experiment $(\mathrm{N}=1,200$ participants from $\mathrm{UK})$ used the same material, but participants were asked whether they would share or not the content in two conditions: in a large anonymous social network, or with their friends, in their favourite social network.

Results: Negative content was both better transmitted in transmission chain experiments and shared more than its neutral counterpart. Threat-related information was successful in transmission chain experiments but not when sharing, and, finally, information eliciting disgust was not advantaged in either.

Conclusions: Overall, the results present a composite picture, suggesting that the interactions between the specific content and the medium of transmission are important and, possibly, that content biases are stronger when memorisation and reproduction are involved in the transmission - like in oral transmission - than when they are not - like in online sharing. Negative content seems to be reliably favoured in both modalities of transmission.

\section{Keywords:}

Cultural transmission; cultural evolution; transmission chain experiments; cultural attraction; content biases; digital media. 


\section{Funding:}

This work was supported by a BRIEF grant provided by Brunel University London.

\section{Conflicts of interest/Competing interests:}

No conflict of interest.

\section{Availability of data and material:}

All anonymised data and material to reproduce the results described in the manuscript are available in an OSF repository online at: https://osf.io/5yh4u/

The experiments were fully preregistered. Preregistration available at: https://osf.io/wf7pd

\section{Code availability:}

The code to analyse the data is available in an OSF repository online at: https://osf.io/5yh4u/

\section{Ethics approval:}

Ethics approval for the study was granted by the College of Health, Medicine and Life Sciences Research Ethics Committee of Brunel University London (ref: 24117-MHRSep/2020-27910-2). 


\section{Introduction}

Evolutionary approaches to culture are used to shed light on contemporary cultural dynamics, such as the evolution of programming languages (Valverde \& Solé, 2015), the prevalence of certain narrative techniques in films (Sobchuk \& Tinits, 2020), the diffusion of music sampling traditions (Youngblood, 2019), or the cognitive underpinnings of vaccine hesitancy (Miton \& Mercier, 2015).

The toolbox of cultural evolution researchers includes transmission chain experiments (Mesoudi \& Whiten, 2008). Transmission chains experiments are a laboratory analogous of the broken telephone game: in a typical set-up, a first participant is presented with a piece of information, often a short story, and is asked to repeat the story to a second participant, which, in turn, will repeat it to a third, and so on, until the last member of the chain. In this way, it is possible to track the transformations that the story undergoes, which new details are added and, mostly, which details are lost or survive through the chain.

Transmission chain experiments often highlight the presence of cognitive biases (Stubbersfield et al., 2017b) or cognitive factors of attraction (Scott-Phillips et al., 2018): certain types of information, possibly because of general evolved cognitive preferences, are more appealing or memorable and tend to be retained and transmitted with more success than others (Sperber \& Hirschfeld, 2004). As an illustration, when presented with a story containing both positive and negative elements, participants tended to remember and transmit preferentially negative elements, suggesting a bias towards negative information (Bebbington et al., 2017).

The same cognitive preferences that influence loss and retention in transmission chain experiments are likely to have an aggregate effect on population-level, real-life, cultural dynamics. The cultural success of a maladaptive practice such as bloodletting, for example, has been linked to its cognitive attractiveness, and transmission chain experiments showed that, when presented with vignette stories with bloodletting or with an alternative therapeutic practice, the bloodletting version tended to be transmitted with more success (Miton et al., 2015). Cognitive biases highlighted in transmission chain experiments have been explored in domains such as urban legends (Stubbersfield et al., 2017a) or online misinformation (Acerbi, 2019b). A negative bias may contribute to explain a decrease in positive emotionality in English language song lyrics in the last 50 years (Brand et al., 2019) or in Anglophone literary fiction in the last two centuries (Morin \& Acerbi, 2017). 
However, the extension from transmission chain experiments to real-life cultural dynamics is not free from issues. If we zoom into the process of cultural transmission, the usual transmission chain set-up resembles oral transmission, where individuals need to pay attention to the information they are exposed to, understand it, memorise it, and then reproduce it to other individuals with communicative intent. (A difference, with respect to oral transmission, is instead that, in the experiments, individuals generally do not choose whether to transmit a piece of information or not.) This contrasts with many instances of real-life cultural transmission. In the cases of music and literature mentioned above, usually individuals do not need to memorise the content they intend to transmit, and intentional modifications are often constitutive part of the process. Even starker is the contrast with digitally mediated transmission, a prominent contemporary example. An online sharing does not require memorisation or reproduction and, in fact, not necessarily understanding, but only the willingness to transmit the content (Acerbi, 2019a).

It is then a critical question how we can generalise the results of transmission chain experiments to real-life cultural domains, or, more broadly, whether and how the details of the process of cultural transmission influence the content that is more likely to spread. Within cultural evolution research, the experiments described in Eriksson \& Coultas (2014) are the first transmission chains that explicitly distinguished three separate phases of transmission: choose-to-receive (do participants want to read a story or not?), encode-and-retrieve (the standard transmission chain procedure) and, finally, choose-to-transmit (do participants want to transmit the story they read or not?). The results showed that the content the experiments were focusing on, that is, content eliciting disgust, was more successful in all three phases. Stubbersfield et al. (2015) compared the performance of social information and survival information in the same three phases of transmission, showing that social information was advantaged over survival information only in the encode-and-retrieve phase, but not in the other two. van Leeuwen et al. (2018) focused on the choose-to-transmit phase, finding limited support both for a negative bias and for an advantage of information eliciting emotions in general. Finally, Stubbersfield et al. (2018) used a modified transmission chain set-up, where participants did not need to recall the story, but were invited to modify it to make it more appealing to the successive readers.

The experiments presented here aimed at comparing explicitly the same material in a standard transmission chain experiment, involving only the encode-and-retrieve phase, with a modality of transmission inspired by online sharing, involving only the choose-to-transmit phase. The experiments investigated three specific content biases that were found successful in 
previous research using transmission chains: negative content (Bebbington et al., 2017), threatrelated information (Blaine \& Boyer, 2018), and information eliciting disgust (Eriksson \& Coultas, 2014).

A negativity bias is consistent with a broad evolutionary logic that would advantage negative information in salience and memorability, as associated to the avoidance of possible dangers (Baumeister et al., 2001). This is in agreement with previous research that found a bias toward negative sentiments in recall in transmission chain experiments (Bebbington et al., 2017), or in acceptance of information (Fessler et al., 2014), and it has been suggested that could influence the diffusion of information online as well (Acerbi, 2019b; Bellovary et al., 2021; Melumad et al., 2021; Schöne et al., 2021). According to the same line of reasoning, it has been proposed that the information being advantaged would not be generally negative information, but a specific subset of it, that is, information concerning possible threats (Blaine $\&$ Boyer, 2018). Finally, an adaptation to prevent disease can explain the salience of stimuli eliciting disgust (Curtis et al., 2004). Transmission chain experiments found that narratives with disgusting elements were better remembered and transmitted than corresponding vignettes without those elements (Eriksson \& Coultas, 2014). Disgust-eliciting particulars have been linked to the success of material such as urban legends (Heat et al., 2001; Stubbersfield et al., 2017a) or European etiquette norms (Nichols, 2002).

The first experiment reproduced an online version of the standard transmission chain setup. For each content bias, I compared the proportion of information successfully transmitted in two chains: one with a version of the story containing the attractive information, and one without. In the second experiment, other participants were instead asked whether they would have shared the same story (either in the attractive or neutral version) in two conditions: with their friends in their favourite social media, or anonymously in a large social media. These two conditions capture the important difference between anonymous and non-anonymous online behaviour (Bernstein et al., 2011). The kind of information shared online, and their tone, are influenced by whether they are shared anonymously or not (Correa et al., 2015). Many reasons can determine this difference, including that anonymous sharing does not affect the reputation of the individuals involved (Boyer, 2018).

The results showed that negative content and threat-related information were, as predicted, favoured in the standard transmission chain setup, but not, surprisingly, information eliciting disgust. Negative information was also favoured when participants were asked to share it (both when sharing with friends and in the anonymous condition), but threat-related 
information was not. Information eliciting disgust, finally, was not favoured in the sharing experiment, consistently with the transmission chain outcome.

The analyses of both experiment 1 and experiment 2 are conducted on data pulled together from two repetitions of the same pre-registered experiments. In Supplementary Information (section 4) I detail the reasons for this choice, and I present separately the results of the originals and the (exact) replications. Ethics approval for the study was granted by the College of Health, Medicine and Life Sciences Research Ethics Committee of Brunel University London (ref: 24117-MHR-Sep/2020-27910-2). The two experiments were fully preregistered at https://osf.io/wf7pd. All data and code to reproduce the analysis and the figures presented here can be found at https://osf.io/5yh4u/.

\section{Experiment 1}

\section{Materials and methods}

For the first experiment, 1,080 participants from UK were recruited online through Prolific $(60 \%$ females, mean age $=36.2, \mathrm{SD}=12.6)$. Participants were pre-screened for being more than 18 years old, for reporting English as their first language, and for using a tablet or a computer desktop (not a mobile phone). Each participant was paid $0.48 £$, or $9.60 £ /$ hour for an estimated completion time of 3 minutes.

For each type of content bias (negative, disgust, threat), 120 independent chains of transmission were run, each including three participants. Three iterations are standard in transmission chain experiments, and it is considered sufficient to reveal the effect of content biases (see e.g., Stubbersfield et al., 2015). 60 chains involved the content where the content bias was present, or attractive content, and 60 involved the content where it was not, or neutral content.

Each story was a short text of five or six sentences. Attractive and neutral contents were represented by the same story, with only one detail changed. For content eliciting disgust, for example, the story involved an outbreak of an infectious disease in the fictional Saint Rika hospital in the US, which was the largest in the last 25 years, and of which 500 cases were identified. In the attractive version, it was reported the information that "the likely source of the outbreak is contact with contaminated faeces in the hospital's toilets," while in the neutral was written that the disease could "be transmitted when a person touches another one." For all 
three stories, both the attractive and neutral variant can be found in Supplementary Information, section 1.

For each chain of transmission, the first participant read the original text and, when ready, they were asked, on a new screen, "to rewrite the story as they were retelling it to a friend." The text generated was then provided to the second participant, and the procedure repeated by passing the text to the third and last participant. The experiment was realised with the software Qualtrics.

The text produced by the participants at each step of the chain was analysed by two coders (the author and an independent coder unaware of the experimental procedure and of the predictions) for half of the chains, and by one coder (the author) for the other half. The coding consisted in determining the presence or absence of basic information from the original story. For content eliciting disgust, for example, were considered (i) the name of the disease, (ii) that the story took place in the US, (iii) in a hospital, (iv) the name of the hospital, (v) that the outbreak was the largest in X (any number of) years, (vi) the correct number of years, (vii) that $X$ (any number of) cases were identified, (viii) the correct number of cases, and (ix) the likely source of the outbreak (or the mean of transmission, for the neutral variant). Between nine and eleven pieces of information were considered for each variant for the three content biases. Complete lists can be found at: https://osf.io/5yh4u/.

Empty texts or texts clearly not related to the task (e.g., participants writing "I could not read the story") were excluded, and participants were replaced. For each of the three content biases, the output, consisting in the proportion of information transmitted at each step of the chain, was analysed using generalised linear mixed models, with the position in the chain and content (attractive/neutral) as fixed effects, and each chain ID and repetition (original/replication) as random intercepts. The analysis was performed with the software $\mathrm{R}$, using the lme4 package (Bates et al., 2015). The prediction was that, for each content bias, the attractive content would be transmitted better than its neutral counterpart.

\section{$\underline{\text { Results }}$}

Overall, for experiment 1, inter-coder agreement was high (94\% probability of agreement, Cohen's $\kappa=0.879)$. As expected, the proportion of content retained and transmitted decreased in all transmission chains (see Fig. 1). The attractive content was better transmitted than the neutral content, confirming the predictions, for negative information $(\beta=0.126, p<0.001)$ and 
threat-related information $(\beta=0.098, p<0.001)$, but there was no difference for information eliciting disgust $(\beta=0.040, p=0.189)$.

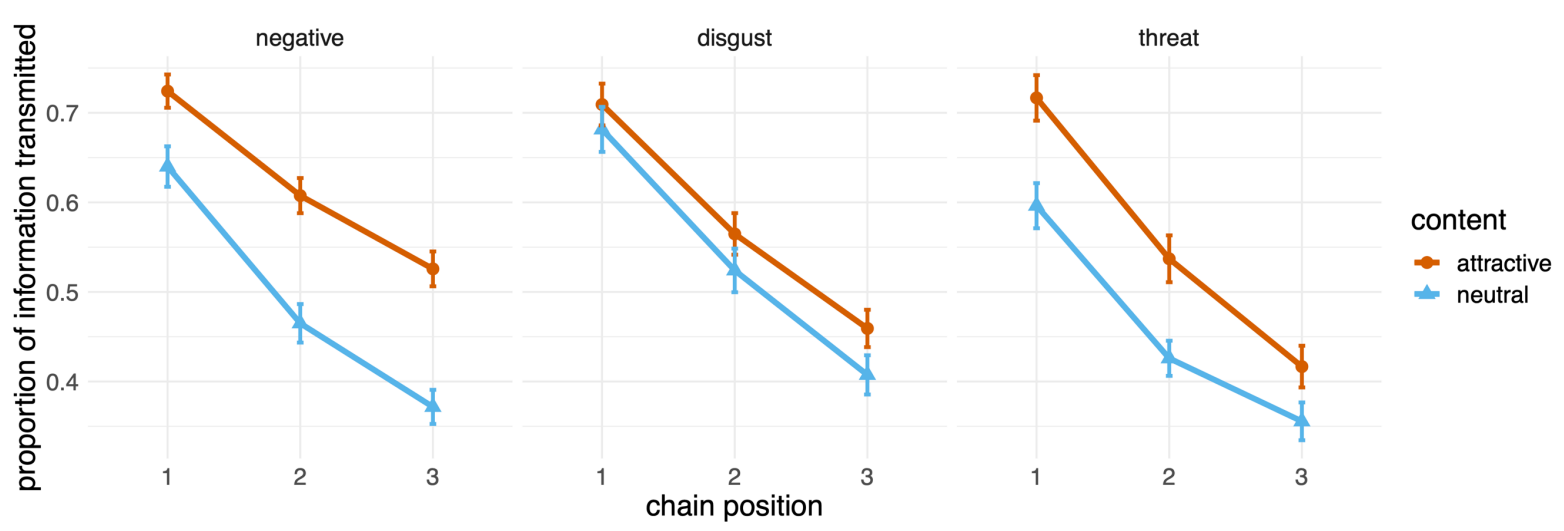

Fig. 1 Proportion of information transmitted for the three content biases in the transmission chain set-up of Experiment 1 (Left: Negative content. Centre: Disgust-eliciting content; Right: Threat-related content). Points indicate the means, and error bars indicate standard errors

\section{Experiment 2}

\section{Materials and methods}

The second experiment involved 1,200 participants from UK, recruited online through Prolific, 600 participants for condition $1(69 \%$ females, mean age $=35.82, \mathrm{SD}=11.4)$, and 600 participants for condition $2(69 \%$ females, mean age $=34.58, \mathrm{SD}=11.4)$. Participants were pre-screened for being more than 18 years old and for reporting English as their first language (the usage of a mobile phone was allowed in this case, as participants did not have to produce written text). Each participant was paid $0.73 £$, or $8.76 £ /$ hour for an estimated completion time of 5 minutes.

Experiment 2 used exactly the same material used as seeds for the transmission chains of experiment 1. In this case, however, the three texts, one for each content bias, were presented to each participant, in random order. For each content bias, again randomly, either the attractive or the neutral version was presented. In condition 1 ("anonymous"), for each text, participants were asked if they would share the story "anonymously, in a large social network, such as Reddit." In condition 2 ("sharing with friends"), the participant was asked if they would share the story "with your friends in your favourite social media." The experiment was also realised with the software Qualtrics. 
The output for each content bias, consisting of the decision of sharing or not, was analysed using generalised linear mixed models (binomial) with content (attractive/neutral) as fixed effect and order of presentation and repetition (original/replication) as random intercepts. As for experiment 1 , the analysis was performed with the software $\mathrm{R}$, using the lme 4 package (Bates et al., 2015). In this case, there was no specific prediction, but the research question was whether in each condition (anonymous/sharing with friends), and for each content bias, the attractive content was shared more than the neutral content.

\section{$\underline{\text { Results }}$}

In experiment 2 (see Fig. 2), the attractive content was shared more than the neutral counterpart for only one content-bias, i.e., negative content, both in the anonymous $(\beta=0.644, p<0.001)$ and in the "sharing with friends" condition $(\beta=0.607, p=0.010)$.

The difference was also significant for information eliciting disgust in the anonymous condition, but in the unexpected direction, with neutral content shared more than attractive content $(\beta=-0.370, p=0.046)$, while no significant difference was found in the "sharing with friends" condition $(\beta=-0.061, p=0.769)$. Finally, no effect was found for threat-related information either in the anonymous $(\beta=0.231, p=0.213)$ or "sharing with friends" condition $(\beta=0.165, p=0.428)$. 


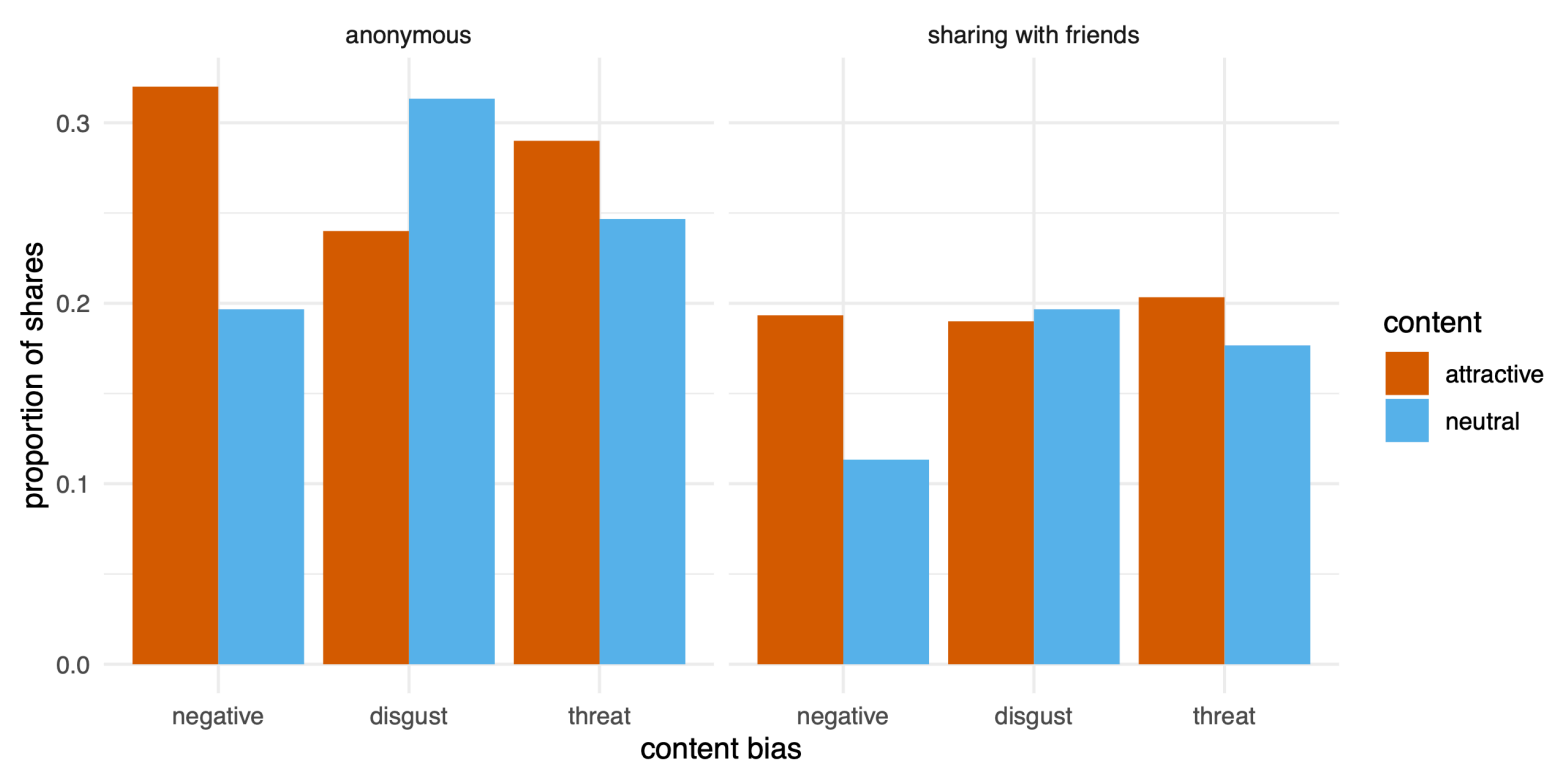

Fig. 2 Proportion of shares for the two conditions of Experiment 2, for the three content biases (Left: "anonymous sharing" condition; Right: "sharing with friends" condition)

\section{Discussion}

As mentioned, the results provided a somewhat composite picture. Comparing the outcomes of experiment 1 (resembling oral transmission) and experiment 2 (resembling online sharing), they were consistent for negative information and disgust-eliciting information, with the former being advantaged, and the latter not, in both experiments. Threat-related information, instead, was advantaged in transmission chain experiments, but not in the sharing condition.

The clearest result is the advantage of negative information both in standard transmission chains and in the sharing experiments, consistent with previous literature showing a ubiquitous negative bias (discussed in the Introduction). On the other side, the present experiments compared a negative narrative with a neutral one, without considering explicitly a positive condition. Other transmission chain experiments found indeed an advantage for emotional content in general (Stubbersfield et al., 2017b) and, as above, emotional content, independently from the direction (positive or negative) has been found favouring online diffusion of content (Berger \& Milkman, 2012; Brady et al., 2017).

Surprisingly, content eliciting disgust was not transmitted or shared differently from its neutral counterpart. In fact, in the anonymous sharing condition of experiment 2 , the attractive content was transmitted less than the corresponding neutral content. This result suggests that fine-grained details of the transmission process may be important for the resulting cultural 
dynamic. For example, we may be attracted by particular content, and perhaps remember it better, but we may not be willing to share it (an obvious example is sex-related information: Berriche \& Altay, 2020). Similarly, subtle cues in the experimental procedure may favour or not the repetition of sensitive material, like the disgust-eliciting details, such as the perception of anonymity, whether participants think that their text will be passed to others, and so on. Another option is that both versions of the story involved a scenario presenting a risk of exposure to pathogens, so they were both attractive, not because of eliciting disgust, but because of the activation of our behavioural immune system. Still, even according to this interpretation, stimuli eliciting disgust should be particularly relevant for the behavioural immune system (Curtis et al., 2011) and possibly resulting advantaged.

Threat-related content was the only content bias for which a difference between the transmission chain experiments and the sharing experiments (in both conditions) was found, with the attractive content being advantaged in the transmission chains but not in the sharing scenario.

The overall contrast between transmitting and sharing, with respect to content biases, is not straightforward. A possible suggestion, worth exploring in future studies, is that the effect of content biases is stronger when retelling a story than when sharing it. The attractive content was favoured in two cases out of three in the transmission chain experiments (negative and threat-related content) and two out of six possible combinations bias/condition in the sharing experiments (negative content on both anonymous and sharing conditions). This difference is clearer when considering the repetitions of the experiments (original and replication) separately (see Supplementary Information, section 4). In this case, attractive content was advantaged four times out of six in the transmission chain experiments and only in two out of twelve possible cases in the sharing experiments. This difference would be consistent with the idea that cognitive factors related to memorisation and reproduction influence the content biases, so that they would be stronger when the medium of transmission requires these phases. More counterintuitively, it would suggest that online sharing may be less content-biased than oral transmission. This may even contribute, together with other reasons, to explain the "paradox" (Altay et al., 2020) for which fake news, despite being cognitively attractive (Acerbi 2019b), have a limited diffusion online (see e.g., Guess et al., 2019; Allen et al., 2020).

Regarding the difference between anonymous and non-anonymous sharing, the effect of the attractive content was not evident. In the case of negative content, the only one in which attractive content was favoured, it was favoured in both conditions. The main difference was that sharing was, in general, less common in the "sharing with friends" than in the "anonymous 
sharing" condition. In the former, the total sharing was around two-third of the latter (322 versus 482). It is also interesting to notice that the total amount of sharing was less than expected, as, overall, the possible occasions to share were 1,800 for each condition, so that participants shared less than $20 \%$ and $30 \%$ of the times, respectively. This may be explained by the fact that stories were possibly interesting but without a personal relevance for participants, and this consideration would be even more important in the "sharing with friends" condition, were reputational concerns could have been involved (Altay et al., 2020).

Some of these results may be explained by the limitations of the current study. First, participants were not actually sharing content in social media (and, as a consequence, they were also not anonymous or not), but they were simply asked if they would share that content. Even though self-reported sharing intentions has been shown to be relatively good indicators of real online sharing behaviour (Mosleh et al., 2020), subtle differences could change the outcomes presented here. Second, small sharing advantages for a type of content could be amplified in real social media by actual sharing. Individuals would then encounter more of the attractive versions, while here they were presented at the same rate. A possible extension that addresses these shortcomings may be represented by digital field experiments, such as having a bot posting attractive/neutral material (of various contents) in real social media and see how the posts are shared.

In the analysis of Experiment 1, consistently with transmission chain studies in cultural evolution literature (e.g., Mesoudi et al., 2006; Stubbersfield et al., 2015), I chose to consider the recall and transmission of information of the whole story presented to participants. The assumption is that the presence of a piece of attractive information would make the whole story more attractive. An alternative analytical strategy would be to consider the performance on only the key pieces of information that distinguish the two versions of the stories. For example, for the content eliciting disgust they would be the reference to faeces/toilets as the source of the outbreak and the reference to touching another person as the mean of transmission, for the attractive and the neutral version, respectively. In Supplementary Information (section 3), I report these results. They confirm the main outcome of negative information being better transmitted than its neutral counterpart, but the effect was reversed for the other two contents, with an advantage for the content eliciting disgust, consistent with previous literature, but no effect for threat-related information. Few studies have explicitly considered how the presence of attractive information influences the performance of the associated (not attractive by itself) information (see e.g., Mermelstein et al., 2021). This, and how different analytical strategies 
related to this difference can influence the interpretation of the results, are important questions for future transmission chain studies.

Another aspect concerns possible differences between the stories presented to participants. Stories used in transmission chain experiments labelled as, say, "negative" are different from each other, making comparisons difficult. Even more, comparing the effect of different content biases, like in the experiments reported here, risks to obscure effects specific to the stories. While that the attractive versions were different from the neutral ones was controlled for (see Supplementary Information, section 2), it is not impossible that the difference between content biases could be due to the differences in how more attractive one story with respect to its counterpart was. For example, the difference in rating between the attractive version and the neutral version of the negative story was stronger than for the two other content biases, possibly contributing to the overall result of negative information being advantaged in both experiments. As for the previous point, systematic transmission chain studies comparing both different narratives within the same content bias and different content biases could be useful to make progress in this direction.

More generally, these experiments point to the importance of keeping into account the details of the transmission process when considering which traits will be favoured by cultural evolution. If content-biases such as the ones studied here can make a cultural trait appealing and memorable, the decision of transmitting it can be detached from these qualities. The types of news that people tend to read most are in general different from the types of news they share most (Bright, 2016). Sharing can be motivated by various interests, including managing one's reputation (Altay et al., 2020) and signalling group membership (Osmundsen et al., 2021), that are not necessarily aligned with the intrinsic attractiveness of a piece of information. Different contents can have different advantages depending on different intentions and modalities of transmission. Ultimately, cultural evolution has mostly focused on consumers of cultural traits as a determinant of cultural success - what they want to copy, from whom-while less attention has been given to the motivation of the transmitters or producers (André et al., 2020). This may be a fruitful direction for future studies.

\title{
Acknowledgments
}

\author{
REDACTED
}




\section{References}

Acerbi, A. (2019a). Cultural evolution in the digital age. Oxford University Press.

Acerbi, A. (2019b). Cognitive attraction and online misinformation. Palgrave

Communications, 5, 15. https://doi.org/10.1057/s41599-019-0224-y

Allen, J., Howland, B., Mobius, M., Rothschild, D., \& Watts, D. J. (2020). Evaluating the fake news problem at the scale of the information ecosystem. Science Advances, 6(14), eaay3539. https://doi.org/10.1126/sciadv.aay3539

Altay, S., Hacquin, A.-S., \& Mercier, H. (2020). Why do so few people share fake news? It hurts their reputation. New Media \& Society, 146144482096989.

https://doi.org/10.1177/1461444820969893

André, J.-B., Baumard, N., \& Boyer, P. (2020). The mystery of symbolic culture: What fitness costs? What fitness benefits? http://dx.doi.org/10.31219/osf.io/kdh $7 \mathrm{t}$

Bates, D., Mächler, M., Bolker, B., \& Walker, S. (2015). Fitting Linear Mixed-Effects Models Using lme4. Journal of Statistical Software, 67(1), 1-48.

https://doi.org/10.18637/jss.v067.i01

Baumeister, R. F., Bratslavsky, E., Finkenauer, C., \& Vohs, K. D. (2001). Bad is Stronger than Good. Review of General Psychology, 5(4), 323-370. https://doi.org/10.1037/10892680.5.4.323

Bebbington, K., MacLeod, C., Ellison, T. M., \& Fay, N. (2017). The sky is falling: evidence of a negativity bias in the social transmission of information. Evolution and Human Behavior, 38(1), 92-101. https://doi.org/10.1016/j.evolhumbehav.2016.07.004

Bellovary, A. K., Young, N. A., \& Goldenberg, A. (2021). Left- and Right-Leaning News Organizations Use Negative Emotional Content and Elicit User Engagement Similarly. Affective Science. https://doi.org/10.1007/s42761-021-00046-w

Berger, J., \& Milkman, K. L. (2012). What Makes Online Content Viral? Journal of Marketing Research, 49(2), 192-205. https://doi.org/10.1509/jmr.10.0353

Bernstein, M., Monroy-Hernández, A., Harry, D., André, P., Panovich, K., \& Vargas, G. (2011). 4chan and /b/: An Analysis of Anonymity and Ephemerality in a Large Online Community. Proceedings of the International AAAI Conference on Web and Social Media, 5(1). https://ojs.aaai.org/index.php/ICWSM/article/view/14134

Berriche, M., \& Altay, S. (2020). Internet users engage more with phatic posts than with health misinformation on Facebook. Palgrave Communications, 6(1).

https://doi.org/10.1057/s41599-020-0452-1

Blaine, T., \& Boyer, P. (2018). Origins of sinister rumors: A preference for threat-related material in the supply and demand of information. Evolution and Human Behavior, 39(1), 67-75. https://doi.org/10.1016/j.evolhumbehav.2017.10.001

Boyer, P. (2018). Minds make societies: How cognition explains the world humans create. Yale University Press. 
Brady, W. J., Wills, J. A., Jost, J. T., Tucker, J. A., \& Van Bavel, J. J. (2017). Emotion shapes the diffusion of moralized content in social networks. Proceedings of the National Academy of Sciences, 114(28), 7313-7318. https://doi.org/10.1073/pnas.1618923114

Brand, C. O., Acerbi, A., \& Mesoudi, A. (2019). Cultural evolution of emotional expression in 50 years of song lyrics. Evolutionary Human Sciences, 1.

https://doi.org/10.1017/ehs.2019.11

Bright, J. (2016). The Social News Gap: How News Reading and News Sharing Diverge. Journal of Communication, 66(3), 343-365. https://doi.org/10.1111/jcom.12232

Correa, D., Silva, L., Mondal, M., Benevenuto, F., \& Gummadi, K. (2015). The Many Shades of Anonymity: Characterizing Anonymous Social Media Content. Proceedings of the International AAAI Conference on Web and Social Media, 9(1).

https://ojs.aaai.org/index.php/ICWSM/article/view/14635

Curtis, V., Aunger, R., \& Rabie, T. (2004). Evidence that disgust evolved to protect from risk of disease. Proceedings of the Royal Society of London. Series B: Biological Sciences, 271(Suppl 4). https://doi.org/10.1098/rsbl.2003.0144

Curtis, V., de Barra, M. \& Aunger, R. (2011). Disgust as an adaptive system for disease avoidance behaviour. Philosophical Transactions of the Royal Society of London. Series B: Biological Sciences, 366(1563), 389-401. https://10.1098/rstb.2010.0117

Eriksson, K., \& Coultas, J. C. (2014). Corpses, Maggots, Poodles and Rats: Emotional Selection Operating in Three Phases of Cultural Transmission of Urban Legends. Journal of Cognition and Culture, 14(1-2), 1-26. https://doi.org/10.1163/15685373-12342107

Fessler, D. M. T., Pisor, A. C., \& Navarrete, C. D. (2014). Negatively-Biased Credulity and the Cultural Evolution of Beliefs. PLoS ONE, 9(4), e95167.

https://doi.org/10.1371/journal.pone.0095167

Guess, A., Nagler, J., \& Tucker, J. (2019). Less than you think: Prevalence and predictors of fake news dissemination on Facebook. Science Advances, 5(1), eaau4586.

https://doi.org/10.1126/sc iadv.aau4586

Heath, C., Bell, C., \& Sternberg, E. (2001). Emotional selection in memes: The case of urban legends. Journal of Personality and Social Psychology, 81(6), 1028-1041.

https://doi.org/10.1037/0022-3514.81.6.1028

Melumad, S., Meyer, R., \& Kim, Y. D. (2021). The Dynamics of Distortion: How Successive Summarization Alters the Retelling of News. Journal of Marketing Research, 002224372098714. https://doi.org/10.1177/0022243720987147

Mesoudi, A., Whiten, A., \& Dunbar, R. (2006). A bias for social information in human cultural transmission. British journal of psychology, 97(3), 405-423.

https://doi.org/10.1348/000712605X85871

Mesoudi, A., \& Whiten, A. (2008). The multiple roles of cultural transmission experiments in understanding human cultural evolution. Philosophical Transactions of the Royal Society B:

Biological Sciences, 363(1509), 3489-3501. https://doi.org/10.1098/rstb.2008.0129

Mermelstein, S., Barlev, M., \& German, T. C. (2021). She Told Me About a Singing Cactus: Counterintuitive Concepts Are More Accurately Attributed to Their Speakers Than Ordinary 
Concepts. Journal of Experimental Psychology: General, 150(5), 972-982.

https://doi.org/10.1037/xge0000987

Miton, H., Claidière, N., \& Mercier, H. (2015). Universal cognitive mechanisms explain the cultural success of bloodletting. Evolution and Human Behavior, 36(4), 303-312.

https://doi.org/10.1016/j.evolhumbehav.2015.01.003

Miton, H., \& Mercier, H. (2015). Cognitive Obstacles to Pro-Vaccination Beliefs. Trends in Cognitive Sciences, 19(11), 633-636. https://doi.org/10.1016/j.tics.2015.08.007

Morin, O., \& Acerbi, A. (2017). Birth of the cool: a two-centuries decline in emotional expression in Anglophone fiction. Cognition \& Emotion, 31(8), 1663-1675.

https://doi.org/10.1080/02699931.2016.1260528

Mosleh, M., Pennycook, G., \& Rand, D. G. (2020). Self-reported willingness to share political news articles in online surveys correlates with actual sharing on Twitter. PLOS ONE, 15(2), e0228882. https://doi.org/10.1371/journal.pone.0228882

Nicols, S. (2002). On The Genealogy Of Norms: A Case For The Role Of Emotion In Cultural Evolution. Philosophy of Science, 69(2), 234-255. https://doi.org/10.1086/341051

Osmundsen, M., Bor, A., Vahlstrup, P. B., Bechmann, A., \& Petersen, M. B. (2021). Partisan Polarization Is the Primary Psychological Motivation behind Political Fake News Sharing on Twitter. American Political Science Review, 1-17.

https://doi.org/10.1017/s0003055421000290

Schöne, J., Parkinson, B., \& Goldenberg, A. (2021). Negativity spreads more than positivity on twitter after both positive and negative political situations. Affective Science. https://doi.org/10.1007/s42761-021-00057-7

Scott-Phillips, T., Blancke, S., \& Heintz, C. (2018). Four misunderstandings about cultural attraction. Evolutionary Anthropology: Issues, News, and Reviews, 27(4), 162-173.

https://doi.org/10.1002/evan.21716

Sobchuk, O., \& Tinits, P. (2020). Cultural Attraction in Film Evolution: the Case of Anachronies. Journal of Cognition and Culture, 20(3-4), 218-237.

https://doi.org/10.1163/15685373-12340082

Sperber, D., \& Hirschfeld, L. A. (2004). The cognitive foundations of cultural stability and diversity. Trends in Cognitive Sciences, 8(1), 40-46.

https://doi.org/10.1016/j.tics.2003.11.002

Stubbersfield, J. M., Tehrani, J. J., \& Flynn, E. G. (2015). Serial killers, spiders and cybersex: Social and survival information bias in the transmission of urban legends. British Journal of Psychology, 106(2), 288-307. https://doi.org/10.1111/bjop.12073

Stubbersfield, J. M., Flynn, E. G., \& Tehrani, J. J. (2017a) Cognitive Evolution and the Transmission of Popular Narratives: A Literature Review and Application to Urban Legends. Evolutionary Studies in Imaginative Culture, 1(1) 121-136.

https://doi.org/10.26613/esic.1.1.20

Stubbersfield, J. M., Tehrani, J. J., \& Flynn, E. G. (2017b). Chicken tumours and a fishy revenge: Evidence for emotional content bias in the cumulative recall of urban legends. 
Journal of Cognition and Culture, 17(1-2), 12-26. https://doi.org/10.1163/15685373$\underline{12342189}$

Stubbersfield, J. M., Tehrani, J. J., \& Flynn, E. G. (2018). Faking the news : Intentional guided variation reflects cognitive biases in transmission chains without recall. Cultural Science Journal., 10, 54-65. https://doi.org/https://doi.org/10.5334/csci.109

Valverde, S., \& Solé, R. V. (2015). Punctuated equilibrium in the large-scale evolution of programming languages. Journal of The Royal Society Interface, 12(107), 20150249. https://doi.org/10.1098/rsif.2015.0249

van Leeuwen, F., Parren, N., Miton, H., \& Boyer, P. (2018). Individual choose-to-transmit decisions reveal little preference for transmitting negative or high-arousal content. Journal of Cognition and Culture, 18(1-2), 124-153. https://doi.org/10.1163/15685373-12340018

Youngblood, M. (2019). Cultural transmission modes of music sampling traditions remain stable despite delocalization in the digital age. PLOS ONE, 14(2), e 0211860.

https://doi.org/10.1371/journal.pone.0211860 


\title{
Supplementary Information - From storytelling to Facebook. Content biases when retelling or sharing a story
}

\author{
Alberto Acerbi
}

\section{1. Full texts of stories}

\section{Negative}

3 Attractive: A woman from Oregon hit the $\$ 8$ million jackpot at a Lucky Eagle Casino slot machine. 4 Veronica Castillo took her mother to the casino in Rochester, Washington. She put $\$ 100$ in a 5 machine and hit the jackpot. The casino staff came over and told her the machine malfunctioned. 6 The woman attempted a legal battle, but she could not claim her money.

7 Neutral: A woman from Oregon hit the $\$ 8$ million jackpot at a Lucky Eagle Casino slot machine. 8 Veronica Castillo took her mother to the casino in Rochester, Washington. She put $\$ 100$ in a

machine and hit the jackpot. The casino staff came over and congratulated her and her mother. It was the highest win from the opening of the casino.

\section{Eliciting disgust}

Attractive: A major outbreak of salmonella has been reported in the US. The outbreak is the largest in the last 25 years. The outbreak had its origin in Saint Rika's hospital. More than 500 cases were identified in the hospital only. The likely source of the outbreak is contact with contaminated faeces in the hospital's toilets.

Neutral: A major outbreak of chickenpox has been reported in the US. The outbreak is the largest in the last 25 years. The outbreak had its origin in Saint Rika's hospital. More than 500 cases were identified in the hospital only. Chickenpox can be transmitted when a person touches another one. 
Attractive: The FBI reported increases in the violent crime rate between 2017 and 2019. Violent crime includes offences such as rape, robbery and assault. Most of the crimes that are reported to police are not solved. This is based on an FBI measure known as the "clearance rate." In 2018, police nationwide cleared less than half of violent crimes that were reported to them.

Neutral: The FBI reported increases in corporate bribery between 2017 and 2019. Corporate bribery means giving a reward to influence someone's behaviour. Most of the bribery cases that are reported are not solved. This is based on an FBI measure known as the "clearance rate." In 2018, police nationwide cleared less than half of bribery cases that were reported to them. 


\section{Stimuli checks}

To assess if there was a reliable difference between attractive and neutral stories, I tested whether attractive stories were indeed rated as more negative, electing disgust, or concerning threats than their neutral counterparts. A total of 180 participants from UK were recruited online through Prolific. Participants were pre-screened for being more than 18 years old and for reporting English as their first language. Each participant was paid $0.50 £$, or $15.00 £ /$ hour for an estimated completion time of 2 minutes.

Three texts, one for each content bias, were presented to each participant, in random order. For each content bias, again randomly, either the attractive or the neutral version was presented. After reading the story, the participant were asked, depending on the context bias, if they agreed with the statement "The story is negative," or "The story elicits disgust," or "The story concerns possible threats." Participants could answer using a 5-Point Likert Scale, with values (1) Strongly agree; (2) Agree; (3) Neutral; (4) Disagree; (5) Strongly disagree. The procedure, as for the main experiments, was realised with the software Qualtrics.

While to a variable degree among the three content biases (see Figure 1), in all three cases the ratings of the attractive version were significantly different, according to a Mann-Whitney U test, from the ratings of the neutral version: negative content $\left(n_{1}=n_{2}=90, U=380, p<0.001\right)$, information eliciting disgust $\left(n_{1}=n_{2}=90, U=906, p<0.001\right)$, and threat-related information $\left(n_{1}=n_{2}=90, U=2108, p<0.001\right)$.
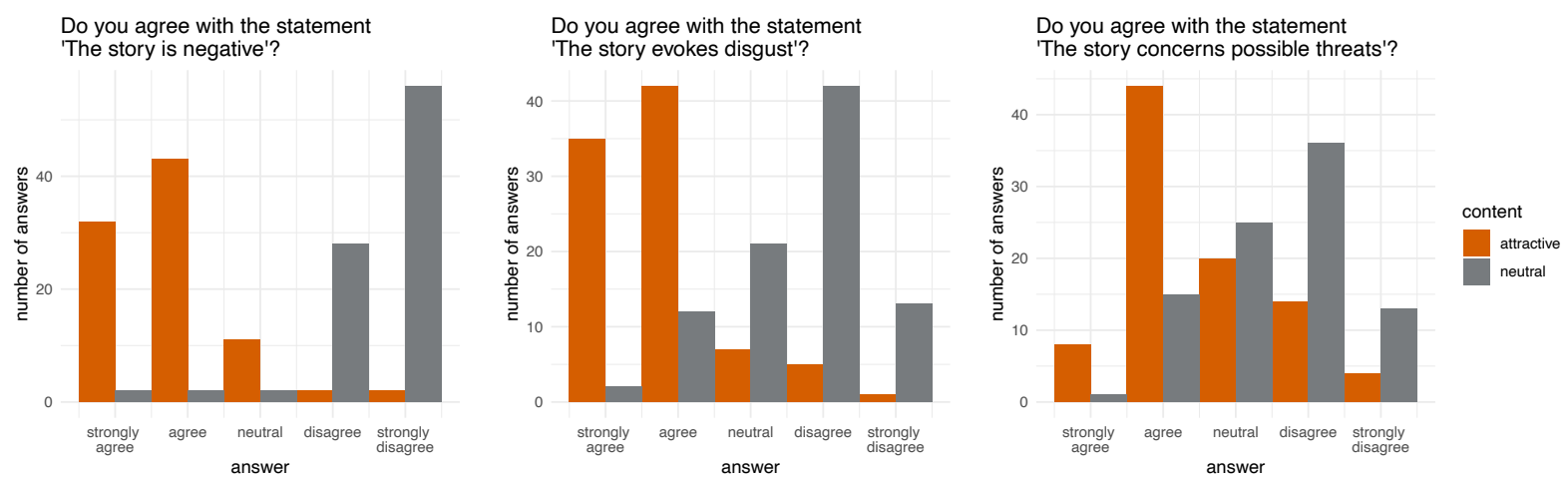

Figure 1: Rating on attractive and neutral stories for the three content biases. 


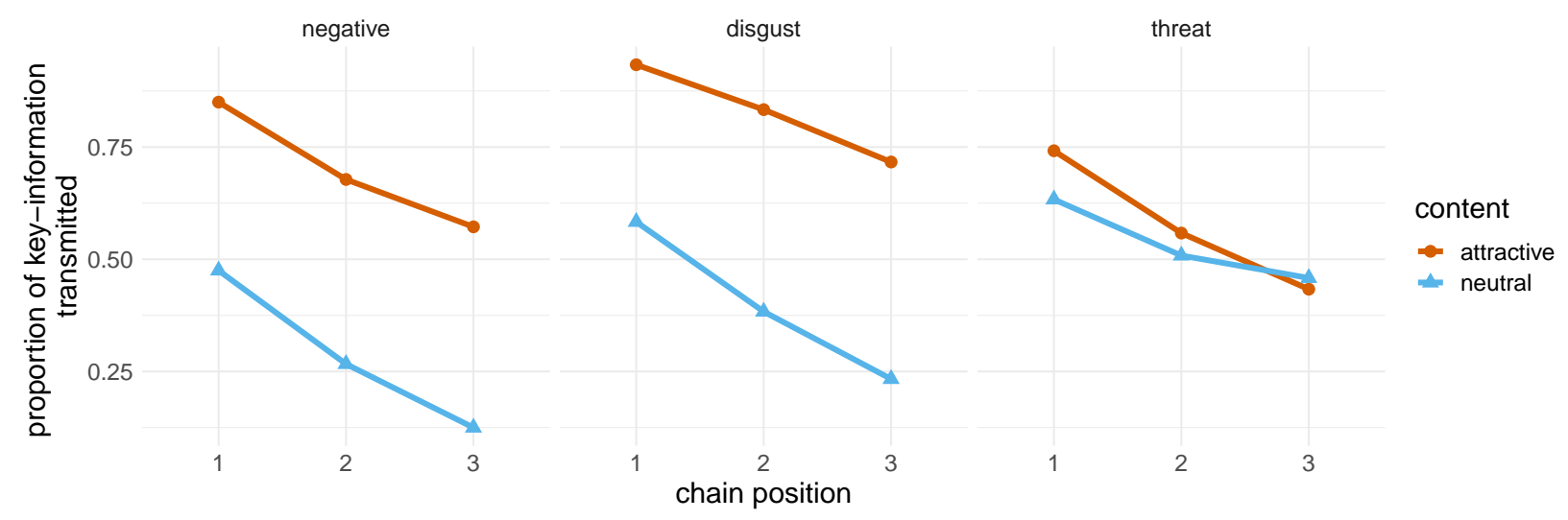

Figure 2: Proportion of key information transmitted for the three content biases in the transmission chain set-up of Experiment 1. 
From a visual inspection (see Figure 2), the output is consistent with the strong effect of negative content, but in this case it seems that there is also a strong effect of the key information on disgust (faeces in the hospital toilets versus a person touching another one) and less effect for the threat-related key information. 


\section{Full results of original experiments and replications}

After running the original experiments, a concern was that the story related to information eliciting disgust, involving an outbreak of an infective disease (see main manuscript), could have evoked associations with the COVID-19 pandemic in the participants, possibly influencing the results. Therefore, I tested two alternative vignettes, inspired by two of the stories used in Eriksson \& Coultas (2014). In both cases, however, the transmission chain experiments did not show that the version with information eliciting disgust was more successful than the alternative (data not shown). Notice this can not be interpreted as a failed replication of Eriksson \& Coultas (2014), as I shortened the stories (to make their length comparable with my material); because my version, differently from Eriksson \& Coultas (2014), was implemented online; and, finally, because I coded the texts differently. In any case, as I become wary of my own results, I decided to replicate my own experiments. As detailed in the manuscript, for the main analysis I pulled together the data of the original experiments and of the replications. Here I report separately the results.

\section{Original experiment}

\section{Experiment 1}

For the first experiment, 540 participants $\left(57 \%\right.$ females, $\left.M_{\text {age }}=36.16, S D=12.8\right)$ were recruited. The methods are as detailed in the main manuscript, with the difference that 60 independent chains of transmission were run for each content bias (30 with the attractive content and 30 with the neutral content), and that the analysis did not include repetition (original/replication) as random effect.

In the original experiment, confirming the predictions, the proportion of content retained remained higher for the attractive content for all three content biases (see Figure 3): negative information $(\beta=0.102, S E=0.034, t(58)=3.010, p=0.004)$, information eliciting disgust $(\beta=0.081, S E=$ $0.039, t(58)=2.065, p=0.043)$, and threat-related information $(\beta=0.145, S E=0.040, t(58)=$ $3.607, p<0.001)$. 


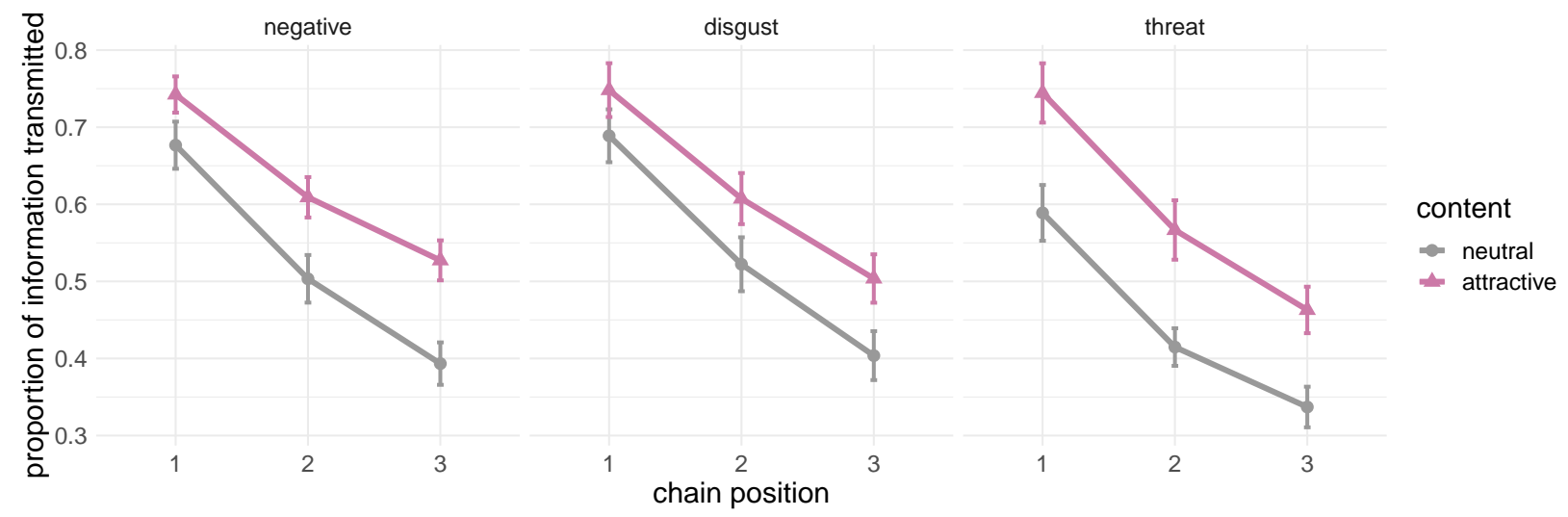

Figure 3: Proportion of information transmitted for the three content biases in the transmission chain set-up of Experiment 1 (original experiment). Points indicate the means, and error bars indicate standard errors.

\section{Experiment 2}

The second experiment involved 600 participants: 300 participants for condition 1 ( $76 \%$ females, $\left.M_{\text {age }}=34.75, S D=11.3\right)$, and 300 participants for condition $2\left(69 \%\right.$ females, $M_{a g e}=34.15, S D=$ 11.1). The methods are as detailed in the main manuscript, with the difference that the analysis did not include repetition (original/replication) as random effect.

The attractive content was shared more than the neutral counterpart in only one out of six possible combinations condition/content-bias, i.e. negative content in the anonymous sharing condition, The full results are as below (see Figure 4). Anonymous sharing condition: negative information $(\beta=0.886, S E=0.280, z(296)=3.167, p=0.002)$, information eliciting disgust $(\beta=-0.270, S E=$ $0.245, z(296)=-1.010, p=0.313)$, and threat-related information $(\beta=0.378, S E=0.270, z(296)=$ $1.402, p=0.161)$. "Sharing with friends" condition: negative information $(\beta=0.180, S E=$ $0.341, z(296)=0.529, p=0.597)$, information eliciting disgust $(\beta=-0.197, S E=0.291, z(296)=$ $-0.677, p=0.498)$, and threat-related information $(\beta=0.077, S E=0.305, z(296)=0.252, p=$ $0.801)$. 


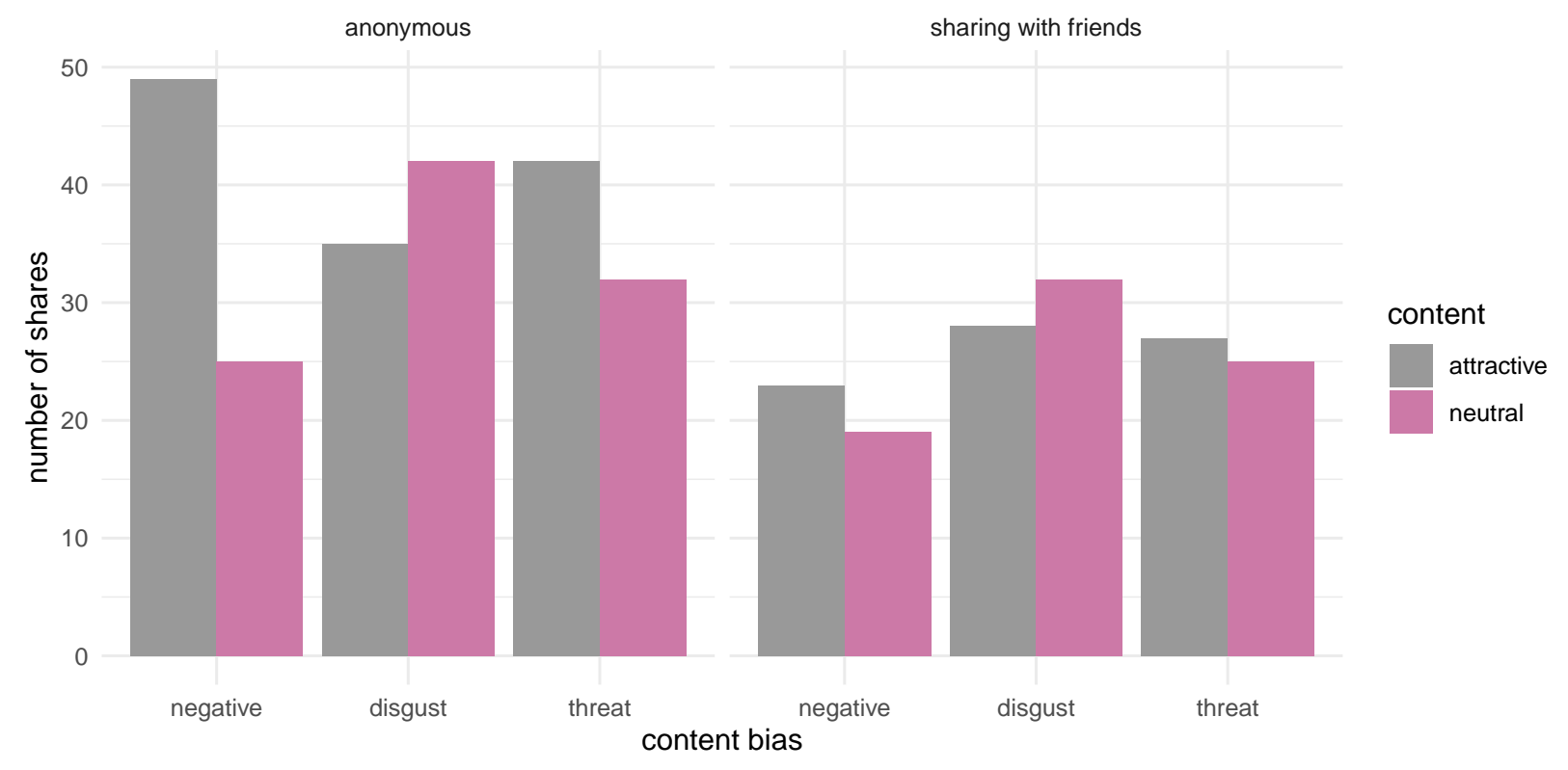

Figure 4: Number of shares for the two conditions of Experiment 2, for the three content biases (original experiment).

\section{Replication}

\section{Experiment 1}

For replication of the first experiment, 540 participants $\left(63 \%\right.$ females, $\left.M_{a g e}=36.47, S D=12.4\right)$ were recruited. As above, the methods are as detailed in the main manuscript, with the difference that 60 independent chains of transmission were run for each content bias, and that the analysis did not include repetition (original/replication) as random effect.

In the replication (see Figure 5), the proportion of content retained remained higher for the attractive content in the case of negative information $(\beta=0.152, S E=0.035, t(58)=4.341, p<0.001)$, but there was no difference for information eliciting disgust $(\beta=-0.001, S E=0.029, t(58)=-0.034, p=$ $0.973)$ and threat-related information $(\beta=0.051, S E=0.031, t(58)=1.303, p=0.198)$.

\section{Experiment 2}

The second experiment involved 600 participants (300 participants for condition 1 (62\% females, $\left.M_{a g e}=36.88, S D=11.5\right)$, and 300 participants for condition $2\left(70 \%\right.$ females, $\left.M_{\text {age }}=35, S D=11.6\right)$. As above, the methods are as detailed in the main manuscript, with the difference that the analysis 


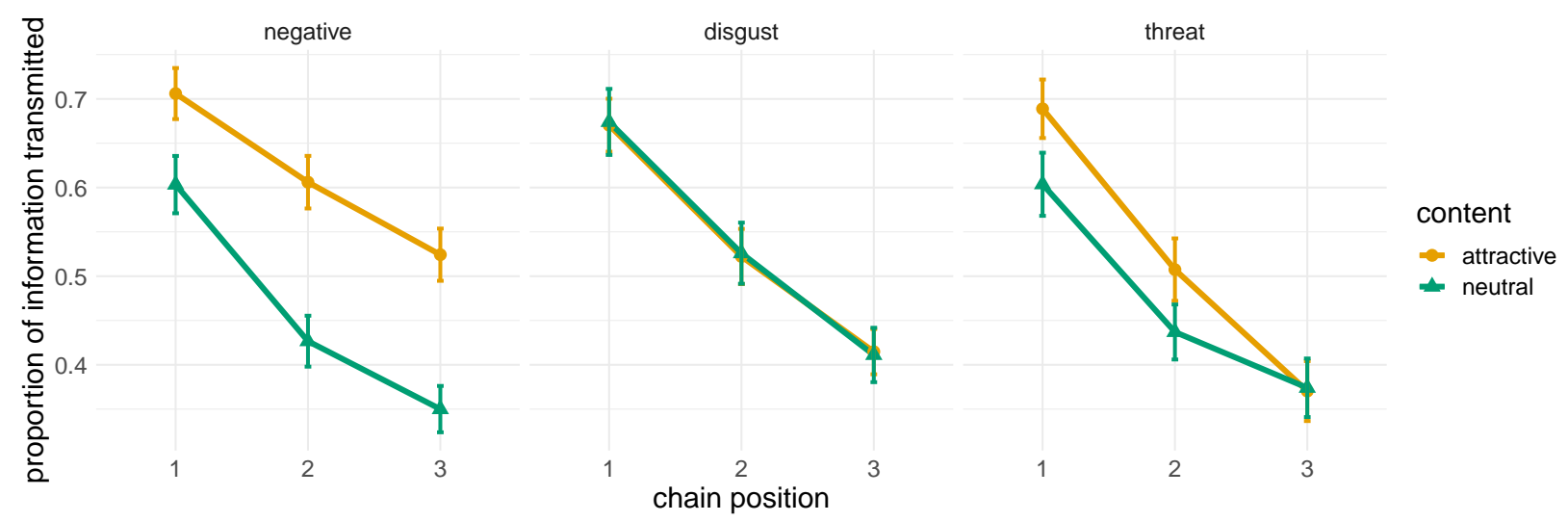

Figure 5: Proportion of information transmitted for the three content biases in the transmission chain set-up of Experiment 1 (replication). Points indicate the means, and error bars indicate standard errors.

did not include repetition (original/replication) as random effect.

In the replication, the attractive content was shared more only in the negative information/sharing with friends case (see Figure 6). The full outputs of the anonymous sharing condition were: negative information $(\beta=0.424, S E=0.263, z(296)=1.615, p=0.106)$, information eliciting disgust $(\beta=-0.452, S E=0.258, z(296)=-1.750, p=0.08)$, and threat-related information $(\beta=0.105, S E=0.256, z(296)=0.410, p=0.682)$. In the "sharing with friends" condition: negative information $(\beta=0.992, S E=0.334, z(296)=2.968, p=0.003)$, information eliciting disgust $(\beta=0.117, S E=0.299, z(296)=0.393, p=0.694)$, and threat-related information $(\beta=0.245, S E=$ $0.286, z(296)=0.854, p=0.393)$. 


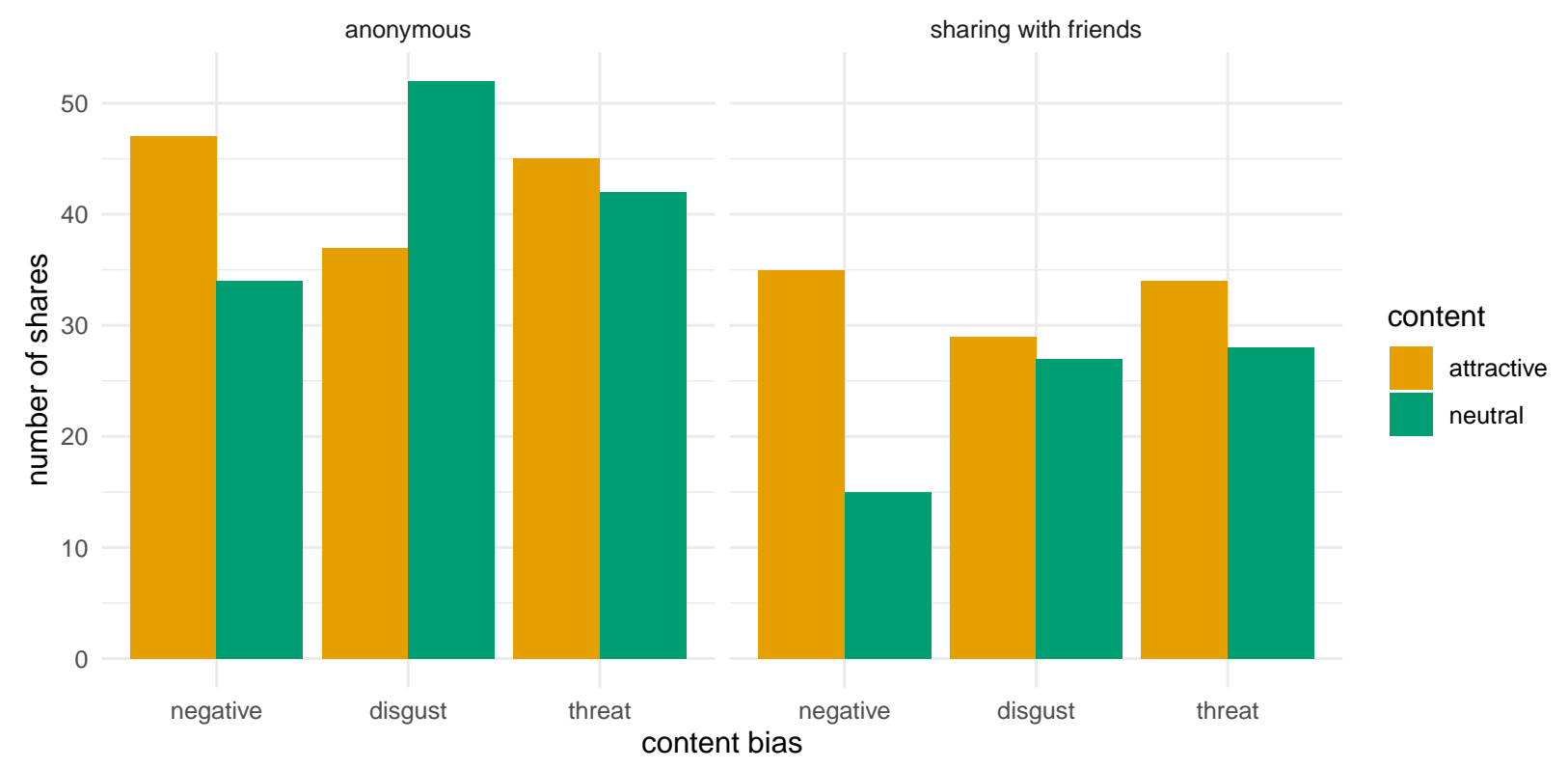

Figure 6: Number of shares for the two conditions of Experiment 2, for the three content biases (replication).

\section{References}

Eriksson, K., \& Coultas, J. C. (2014). Corpses, Maggots, Poodles and Rats: Emotional Selection Operating in Three Phases of Cultural Transmission of Urban Legends. Journal of Cognition and Culture, 14(1-2), 1-26. https://doi.org/10.1163/15685373-12342107 